\title{
The TOPSAR Interferometric Radar Topographic Mapping Instrument
}

\author{
IIOWARI) A. ZkBKER, SøreN N. MADSFN, JaN MARTIN \\ Jet Propulsion Laboratory \\ California Instilute of Technology \\ Glovanni Aiberti and Sergio Vetrella \\ CO.RI.S.T.A. \\ Alissandro CUCCI \\ Alenia S.p.A.
}

\begin{abstract}
We have augmented the $\ \triangle S$ DC-8 AIRSAR instrument with a pair of C-band antennas displaced across track to form an interferometer sensitive to topographic variations of the Earth's surface. The antennas were developed by the Italian consortium Co.Ri.S.T. A., under contract to the Italian Space Agency (ASI), while the AIRSAR instrument and modifications to it supporting TOPSAR were sponsored by NASA. A new data processor was developed at JPL for producing the topographic maps, and a second processor was developed at Co.Ri.S.T.A. All the results presented below were processed at JPL. During the 1991 DC-8 fight campaign, data were acquired over several sites in the United States and Furope, and topographic maps were produced from several of these flight lines. Analysis of the results indicate that statistical errors are in the $2-3 \mathrm{~m}$ range for flat terrain and in the $4-5 \mathrm{~m}$ range for mountainous areas.
\end{abstract}

\section{Introduction}

We have implemented an interforometric synthetic aperture radar system for lopographic mapping applications (TOPSAR) on the NASA DC-8 aircraft. NASA /JPI, currently operates a multifrequency (P, $\mathrm{L}$, and $\mathrm{C}$ bands), multipolarimetric radar (AIRSAR) on board this aircraft. The TOPSAR implementation uses much of the existing $A I R S A R$ hardware, although several modifications were required to optimize performance in the topographic mapping mode. When in use, TOPSAR effectively replaces the C-band polarimeter instrument, but the remaining $\mathrm{I}$ - and $\mathrm{P}$-band systems are undisturbed and operate together with the topographic mapper; therefore the combined instrument produces simultaneous L-and P-band fully polarimetric plus C-band VV polarization backscater images in addition to the topographic product.

In this paper we first describe our implementation, including system design parameters, required modifications to the existing AIRSAR hardware, and new anterna design. We then discuss data processing strategy and display somo example interferometric images. Finally, wo analyze an image acquired over Ft. lrwin, California, in terms of height accuracy.

\section{Background}

Interferometric radar has been been proposed and successfully demonstrated as a topographic mapping technique by Graham [1], Zebker and Goldstein [2], and Gabriel and Goldstein [3]. Two distinct implementation approaches have been discussed for lopographic radar interferometers which differ in how the interferometric baseline is formed. In one case a single antonna and radar system illuminates a given surface at $($ wo different limes but with nearly the same viewing geometry, forming a synthetic interferometer. This case has been implemented both from spaceborno platforms (sce, for example, Goldstein et al., [4]) and also from aircraft (Gray and Farris-Manning [5]). In the second approach the baseline is formed by two physical antemas which illuminate a given area on the ground simultaneously. This is the approach used originally by Craham [1] and also by Zobker and Goldstein [2] for the NASA CV-990 radar; it is the approach used here.

\section{Revicw of theory}

Consider a set of two antemas $A 1$ and $A 2$ as shown in figure 1. The surface topography is given by $z(y)$, 
$\mathrm{h}$ is the aircraft altitude, the baseline distance is $\mathrm{B}$, the range to a point on the ground is $\rho$, the look angle $\theta$, and the angle of the baseline with respect to horizontal is $\alpha$. Radar echoes are transmitted from antenna $A 1$ and received simultaneously at $A 1$ and $A 2$, thus the difference in path lengths is $\delta$, which depends on the baseline distance, baseline angle, range, look angle, and the height of the point $\mathrm{z}(\mathrm{y})$. The measured phase of the interferometer is directly proportional to this distance, with the constant of proportionality $\frac{2 \pi}{\lambda}$. A little algebra and geometry yield the following equations for height as a function of these parameters:

$$
\begin{gathered}
\delta=\frac{\lambda \phi}{2 \pi} \\
\sin (\alpha-\theta)=\frac{(\rho+\delta)^{2}-\rho^{2}-B^{2}}{2 \rho B} \\
z(y)=h-\rho \cos \alpha \cos (\alpha-\theta)-\rho \sin \alpha \sin (\alpha-\theta)
\end{gathered}
$$

where $\phi$ is the measured phase, and $\lambda$ is the wavelength.

Differentiation of (1-3) yields the error in height estimate as a function of the error in phase estimate:

$$
\sigma_{h}=\frac{\lambda \rho}{2 \pi B}[\sin \alpha-\cos \alpha \tan (\alpha-\theta)] \sigma_{\phi}
$$

where $\sigma_{h}$ and $\sigma_{\phi}$ are the standard deviations of height and phase, respectively.

There exists an optimum baseline distance that minimizes the total height error [Rodriguez and Martin, 6]; this optimum is quite broad and for high ( $\geq 10 \mathrm{~dB}$ ) signal to noise ratio systems can be anywhere in the range of $0.2-0.8$ of the critical value which may be calculated by (Zebker and Villasenor [7]):

$$
B_{c}=\frac{\lambda \rho}{R_{y}} \tan \theta
$$

where $R_{y}$ is the slant range resolution. Note that this equation differs from that of Zebker and Villasenor by a factor of $2 \cos \theta \sin \theta$ as they were restricted to horizontal baselines, used the ground rather than slant range resolution, and assumed a single antenna in repeat-track configuration rather than two physical antennas as we use in TOPSAR. Another significant error source results from errors in knowledge of the aircraft attitude, the most important component of which is roll angle, which in our system translates directly to an error in look angle. Again differentiation yields

$$
\sigma_{h}=\rho \sin \theta \sigma_{\theta}
$$

Thus, good motion compensation is a prerequisite of any practical system.

\section{TOPSAR design}

Several TOPSAR system parameters are summarized in Table 1.

Table 1. TOPSAR radar system parameters

\begin{tabular}{ll} 
Parameter & TOPSAR value \\
\hline Peak power, watts & 1000 \\
Pulse rate, $\mathrm{Hz}$ & 600 nominal \\
Pulse length, $\mu$ sec & 5.0 \\
Antenna length, m & 1.6 \\
Antenna width, m & 0.11 \\
Antenna gain, dB & 25 \\
Range bandwidth, MHz & 40 \\
Receiver noise temperature, K & 2100 \\
Antenna baseline, m & 2.58 \\
Baseline angle $(\alpha)$, deg & 62.77
\end{tabular}

Given the baseline separation and the signal to noise ratio we can then analyze performance of the interferometer. Figure 2 ( $\mathrm{Li}$ and Goldstein [8], Zebker and Villasenor [7]) gives the expected phase error as 
a function of signal to noise ratio and number of looks for a radar interferometer. Thus we may expect a phase error of $3.3^{\circ}$ if the data are processed to 16 looks assuming an SNR of $13 \mathrm{~dB}$, which implies a statistical error of $1.50 \mathrm{~m}$ in height precision. Knowledge of the roll angle is also necded for accurate data reduction. At present our system can correct for attitude errors to the $0.015^{\circ}$ level, which (6) implies a height error of $1.9 \mathrm{~m}$.

Figure 3 shows a set of data acquired over Fort Irwin in the Mojave Desert in California. The data shown represent ground coordinate rectified topographic maps in which the color contour interval is $6 \mathrm{~m}$, thus one trip around the color wheel corresponds to $96 \mathrm{~m}$ for our 16 entry color table. The spatial resolution is $10 \mathrm{~m}$. The accuracy of the map obtained over Ft. Irwin is described in the next section.

\section{Verification procedure}

Verification of this mapping technique involves comparing the radar-derived height map with pre-existing high-resolution digital elevation models (DEMs) and characterizing the differences. Comparison was done on a data set from the Ft. Irwin area in California using the USGS 7.5' DEM with $30 \mathrm{~m}$ grid spacing and $7 \mathrm{~m}$ rms height error. Figures 4 shows a sample cut through the DEM and radar-generated terrain maps, and the difference between them is shown in the upper part of the figure. It is clear from examination of this figure that the error is dominated by differences in areas of high relief, and is caused both by errors in overall cross-track slope and by position errors.

\section{References}

[1] Graham, L.C., Synthetic interferometer radar for topographic mapping, Proc. IEEE, vol 62, 763-768, 1974 .

[2] Zebker, H. and R. Goldstein, Topographic Mapping from Interfermetric SAR Observations. Journal of Geophysical Rescarch v.91, No. B5, p. 4993-4999.1986.

[3] Gabricl, A. K. and R. M. Goldstein, Crossed orbit interferometry: theory and experimental results from SIR-B, Int'l Journal Remote Sensing, vol 9., no. 8, pp. 857-872, 1988.

[4] Goldstein, R. M., H. A. Zebker and C. Werner. Satellite radar interferometry: two-dimensional phase: unwrapping, Radioscience, vol. 23, no. 4, pp 713-720, July-August 1988.

[5] Gray, A. Laurence and P. J. Farris-Manning, Two-pass interferometry with airborne synthetic aperture radar, submitted to IEEE Trans. Geo. Rem. Sens., 1991.

[6] Rodriguez, E. and J. Martin, Theory and design of interferometric SARs, submitted to Procecding of the IEE, 1991.

[7] Zebker, H.A. and J. Villasenor, Decorrelation in interferometric radar echoes, submitted to IEEE Trans. Geo, Rem. Sensing, August, 1991.

[8] Li, F. and R. M. Goldstein, Studies of multi-baseline spaceborne interferometric synthetic aperture radars, IEEE Trans. Geo. Rem. Sens., vol. 28, no. 1, pp. 88-97, January 1990.

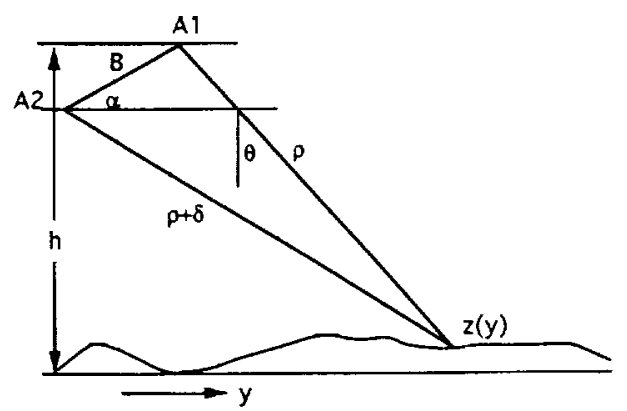

1. TOPSAR geometry. Radar echoes are transmitted from antenna $A 1$ and received simultaneously at $A 1$ and $A 2$. The phase difference of the two echoes is proportional to the difference in path lengths $\delta$, which depends on the baseline distance $B$, baseline angle $\alpha$, range $\rho$, look angle $\theta$, aircraft altitude $\mathrm{h}$, and the height of the point $\mathrm{z}(\mathrm{y})$.

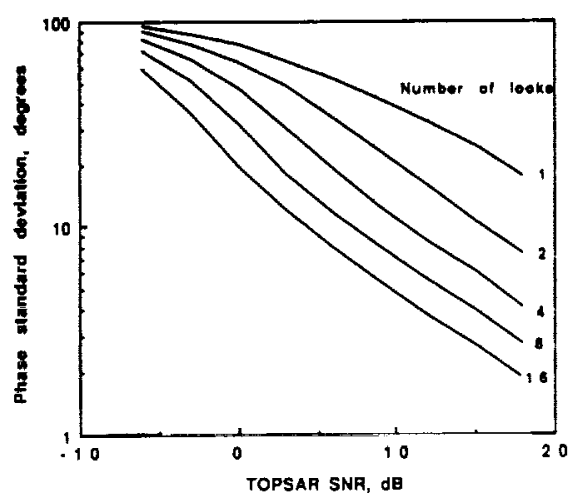

2. Sensitivity of phase standard deviation to signal-to-noise ratio and number of looks in processor. Increasing number of looks is an effective means to reduce statistical variation, especially for the first 8 looks or so. 

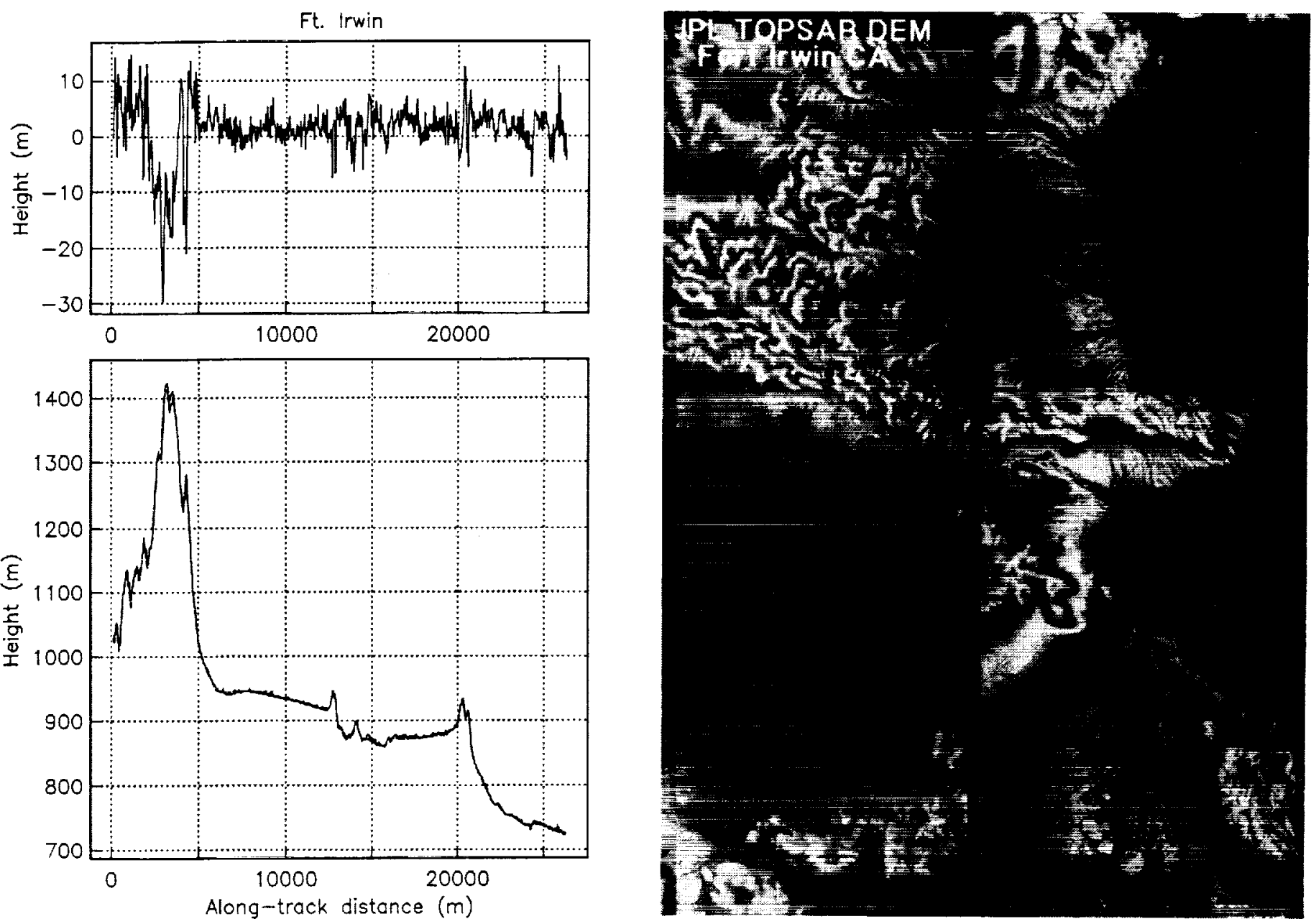

3. Topographic data acquired over Fort Irwin in the Mojave Desert in California. The data shown represent ground coordinate rectified topographic maps in which the color contour interval is $6 \mathrm{~m}$. 